\title{
Contents, Vol. 83, 1934
}

Inhaltsverzeichnis.

Eigenarbeiten. seite

Bariels, M, Entstehung von Dämmerungszittern der Augen

beim Menschen

172

Böck, J. und E. Risak, Periodische Okulomotoriuslähmung

und Tuberkulose 7

Edeskuty, Ø.,ÜbereinerezidivverhütendePterygiumoperation 109

Fischer, F. , Anatomische Befunde zur Behandlung der Netzhautabhebung mit multipler diathermischer Stichelung 95

v. Herrenschwand, F., Die Wundsprengung nach Altersstar-Extraktion und ihre Verhütung durch die Anlegungeines vorbereiteten Bindehautlappens ..... 87

Hesse, R., Zur pathologischen Anatomie der Skleritis . 324

Jess, A., Extraktion eines Röntgenstars. Zysteinreaktion

in der Röntgenstarlinse $\quad 260$

Krassó, I., Die Beurteilung des histologischen Befundes im

zweiten, sympathisch erkrankten Auge jy

Kraupa, E., Über Epithel- und Endotheldystrophien . . 179

Kyńeleis, W., Scheinbare Pupillenstarre (Pupillotonie) mitFehlen von Sehnen- und

Periostreflexen. (Eine System-erkrankung?) 278

Majoros,J., Die Wirkung des intravenös gegebenen Natrium-

salizylat bei Augenkrankheiten

16

Meller, J., Über die bazilläre Ätiologie der Ophthalmiasympathica und mancher Fälle von

Iridocyclitis post-traumatica 145

Mussabeili, U. , Zur pathologischen Anatomie des Buphthalmus 27

Ohm, J., Der Nystagmus bei der totalen Farbenblindheit 1

- Zum Nachweis von Metallsplittern im Körper mit

Hilfe der Elektronenröhre 287

-,-, Der optokinetische Nystagmus bei Septojodschädigung

der Netzhaut 338

Purtscher, A., Tuberkelbazillenkultur aus dem Gewebe des

sympathisierenden Auges 163

Risak, E., siehe Böck, J.

RohrSchneider, W., Die Verbreitung desTrachoms in Deutsch-

land 263

Safáf, K., Behandlung der Netzhautabhebung mit diathermischer Stichelung und deren

Ergebnisse . . . 189

Sallmann, L., Kasuistischer Beitrag zur Frage der bazillären

Natur der sympathischen Ophthalmie . $\quad$.... 168

- IV - 
Scifce

Schieck, F., Hochgradige allergische Phasen im Verlaufe der

Iristuberkulose und ihre Behandlung 257

Schmelzer, H., Über die augendrucksteigernde Wirkungdes Diocains. - Welches Anästhetikum eignet sich ambesten zum Tonometrieren ? 331

Vogt, A., Die Gefahren der Nadeldiathermie 321

,-- , Kathoden-Elektrolyse als neuer Weg zum Auffinden

und zum Verschluß des Netzhautrisses 322

Aus der Praxis - für die Praxis.

Jahnke, W., Ekzem der Lider nach Pantocainbetäubung 112

Mieses-Reif, M., Vereinfachte Fadenlampe 35

Vormann, ].. Beitrag zu dem Behrschen Syndrom der Ver-

änderungen in der Fissura orbitalis superior . . . 288

Berichte über die ophthalmologische Literatur.

Vererbung und Auge. Von Prof. Dr. A. Franceschetti in Genf . . 36 Physikalische Optik. Dioptrik des Auges. Brillenkunde. Unter-

suchungsmethoden zur Refraktionsbestimmung. Von Doz. Dr.

R. Kramer in Wien 52

Ophthalmologische Therapie. I. Medikamentöse Therapie. Von Dr.

E. Kunz in Bern 114

II. Operative Therapie. - III. Physikalische Therapie. -

IV. Strahlentherapie. Von Prof. Dr. W. Hoffmann in Königsberg 119 Entstehung und Klinik der

Refraktionsanomalien, Sehschärfe, Ak-

kommodation. Von Doz. Dr. L. Sallmann in Wien 198

Vegetative Physiologie und Pathologie. Von Priv.-Doz. Dr. W.

Rauh in Gießen 205

Verletzungen. - Versicherungswesen und soziale Medizin. - Blin-

denwesen. Von Dr. R. Heßberg in Essen 219

Bakteriologie, Parasitologie, Immunitätsforschung und Vergiftungen.

Von Dozent Dr. A. Pillat in Wien 294

Kornea, Sklera, Episklera. Von Prof. Dr. H. Schmelzer in Erlangen 343

$\mathrm{G}^{1} / 8$ sellschaftsberichte.

Ophthalmologische Gesellschaft in Wien. Sitzung vom 15. Jan. 193466

Sitzung vom 19. Febr. 1934 23z Sitzung vom 12. März 1934354 Verein rheinisch-westfälischer Augenärzte. Sitzung am 19. November

!933 in Düsseldorf 130

Augenärztliche Vereinigung im Oberbergamtbezirk Dortmund.

Sitzung vom 20. Januar 1934136

Vereinigung mitteldeutscher Augenärzte. 37. Tagung am 9. und

io.Dezember 1933 in Halle a. d. S 236

Bayerische Augenärztliche Vereinigung. Achte Tagung in Erlangen

am 9. und 10. Dezember $1933 \quad 241$

Diagnose und Therapie $\quad 71,139,252,317,364$

Buchbesprechungen 74, 255

Personalien 75, 144, 256, 320, 369

Tagesnaehriehten 144, 320 
S. Sachregister zu Band $83 \quad 370$

Namenregister zu Band $83 \quad 376$ 